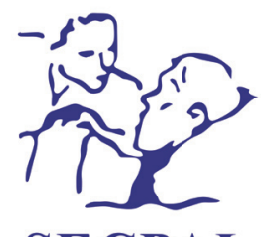

Medicina Paliativa

\title{
Impacto emocional en pacientes y familiares durante la pandemia por COVID-19. Un duelo diferente
}

\author{
María Antonia Lacasta-Reverte ${ }^{1^{*}}$, Marta Torrijos Zarcero², Yolanda López-Pérez ${ }^{3}$, \\ Diego Carracedo Sanchidrián², Teresa Pérez Manrique ${ }^{1}$, Carlos Casado Sanmartín², \\ Cristina Rocamora González ${ }^{4}$, Laura Blanco Rosado², Noelia Iglesias Gutiérrez², \\ Emma Vidal Bermejo², Daniel Trigo Varela² y Jesús Martí-Esquitino²
}

${ }^{1}$ Unidad de Cuidados Paliativos, Hospital Universitario La Paz. Madrid, España. ${ }^{2}$ Servicio de Psiquiatría, Psicología Clínica y

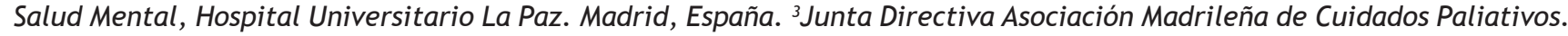
Madrid, España. ${ }^{4}$ Obra social La Caixa. Madrid, España

Recibido el 8 de julio de 2020

Aceptado el 26 de agosto de 2020

\section{PALABRAS CLAVE \\ Duelo, COVID-19, atención psicológica.}

\begin{abstract}
Resumen
Objetivo: Favorecer que cada persona elabore su duelo de forma natural, prevenir factores de riesgo e identificar a los deudos que necesiten una atención específica.

Método: Estudio descriptivo. Doscientas ocho familias fueron atendidas entre el 11 de marzo y el 20 de mayo de 2020 por duelo por la COVID-19, de acuerdo con un protocolo establecido. Resultados: La edad media de los fallecidos fue de 76 años. El 58 \% eran hombres. Los familiares atendidos fueron hijo/a (54\%), cónyuge o pareja (20\%), hermano/a (2 \%). En relación con el lugar de fallecimiento, el $41 \%$ en plantas de hospitalización, el $27 \%$ en Urgencias, el 22 \% en la planta de cuidados paliativos y el $10 \%$ en $\mathrm{UCI}$.

El tipo de atención fue en el $53 \%$ de los casos telefónica, en el $16 \%$ presencial, en el $12 \%$ telefónica y presencial y en el $3 \%$ a través de videollamada. En un $63 \%$ de las veces se realizó apoyo, asesoramiento puntual o atención específica mientras que en el 37 \% se ofreció apoyo y disponibilidad.

En lo referente "despedida final", el $49 \%$ de los familiares pudieron despedirse, el $31 \%$ no pudo. El tipo de duelo de los familiares atendidos fue inicialmente normal en el $71 \%$, mientras que un $16 \%$ fue clasificado como de riesgo.

Conclusiones: La información y comunicación efectiva con los familiares afectados, la formación en la muerte y el duelo de los profesionales implicados y la disponibilidad de atención psicológica se consideran de gran importancia en una situación COVID-19.
\end{abstract}

*Autor para correspondencia: María Antonia Lacasta-Reverte

Unidad de Cuidados Paliativos, Hospital Universitario La Paz. Paseo de la Castellana, 261. 28046, Madrid, España

Correo electrónico: mariantlacasta@yahoo.es 
La experiencia muestra que la "Atención al duelo" debe estar a disposición de la población general afectada. Una buena organización y planificación ha de incorporar protocolos de actuación y seguimiento que incluyan una adecuada valoración y atención en los diferentes niveles desde el apoyo, la prevención y la detección precoz de posibles complicaciones en el duelo, y si es necesario la intervención especializada.

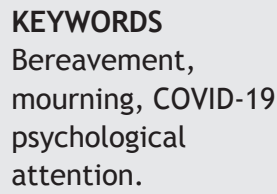

Lacasta-Reverte MA, Torrijos Zarcero M, López-Pérez Y, Carracedo Sanchidrián D, Pérez Manrique T, Casado Sanmartín C, et al. Impacto emocional en pacientes y familiares durante la pandemia por COVID-19. Un duelo diferente. Med Paliat. 2020;27(3):201-208

\begin{abstract}
Objective: To allow each individual to undergo bereavement in a natural way, to prevent risk factors, and to identify bereaved people who need specific attention.

Method: A descriptive study. Two hundred and eight families were attended to between March 11 and May 20, 2020 for Covid-19 according to an established protocol.

Results: Average age of the deceased relatives was 76 years. Fifty-eight percent were men. The family members who were cared for included: son or daughter (54\%), spouse or partner (20\%), brother or sister (2\%). Regarding the place of death, $41 \%$ were in hospitalization wards, $27 \%$ in the emergency room, $22 \%$ in the palliative care ward, and $10 \%$ in the ICU.

The method of care was in $53 \%$ of cases over the telephone, in $16 \%$ in person, and in $12 \%$ both over the telephone and in person; in $3 \%$ of cases it was by video call. In $63 \%$ of cases, support, specific advice or care was provided, whereas in $37 \%$ support and availability were offered.

In terms of "final goodbye", $49 \%$ of all family members were able to say goodbye, while $31 \%$ were unable to do so. The type of grief observed in the family members attended to was initially considered to be normal for $71 \%$ of cases, whereas $16 \%$ were classified as at risk.

Discussion: Effective information to and communication with affected relatives; that involved professionals be trained in dealing with death and grief so as not to cause harm, as well as in palliative care; and availability of psychological care are deemed of great importance in a COVID-19 scenario.

Experience shows that "bereavement care" should be available to the general affected population. Proper organization and planning must incorporate action and follow-up protocols that include adequate assessment and care at different levels, from support, prevention and early detection of possible complications of bereavement to specialist intervention when necessary.
\end{abstract}

\section{INTRODUCCIÓN}

La situación excepcional de COVID-19 y sus consecuencias no previstas han ocasionado un gran impacto emocional en los enfermos, familiares y profesionales ${ }^{1,2}$, situación a la que en la mayoría de los casos se ha unido a un "confinamiento", llevándonos a realizar cambios en todos los ámbitos de nuestra vida ${ }^{3,4}$.

En el caso del fallecimiento a consecuencia de la COVID-19, nos coloca ante un escenario diferente por la rapidez de los acontecimientos, la incertidumbre y la novedad en el proceso al que es necesario atender ${ }^{5-7}$. Esta es la razón por la que la atención al duelo está a disposición de la población general afectada. Una buena organización y planificación ha de incluir una adecuada valoración y atención en los diferentes niveles desde el apoyo, la prevención y la detec- ción precoz de posibles complicaciones en el duelo, y si es necesario la intervención especializada ${ }^{8-11}$. Atender a estas personas es una tarea delicada y de gran importancia para la salud biopsicosocial ${ }^{8,12,13}$.

\section{SITUACIÓN ACTUAL: COVID-19}

\section{COVID-19: un duelo diferente}

La muerte de un ser querido significativo es uno de los acontecimientos vitales más estresantes que ha de afrontar una persona. Sin embargo, el duelo está considerado un proceso normal y cumple una función de adaptación, no requiriendo en la mayoría de los casos intervenciones específicas, ni psicofármacos. 
Se pueden identificar algunos factores circunstanciales en este tipo de duelo que lo hacen diferente con respecto a otros duelos ${ }^{12,14-19}$ (Tabla I).

Estas circunstancias podrían dificultar la elaboración del duelo. Sin embargo, la mayoría de las personas muestran una gran resiliencia ${ }^{14,20,21}$, y tienen la capacidad para hacer frente a la adversidad de forma adecuada. Hay también quien considera que se puede aliviar la vivencia del duelo si la muerte es anticipada y se ha podido acompañar al ser querido durante la enfermedad hasta los últimos momentos de la vida ${ }^{14,15}$.

\section{Factores de riesgo en el duelo por la COVID-19}

Aunque el duelo por la COVID-19 puede cumplir criterios en algunos dolientes para desarrollar complicaciones ${ }^{22-24}$, una atención precoz con objetivos preventivos en personas que presenten factores de riesgo puede facilitar un duelo normal y evitar complejidad. En este nuevo escenario COVID-19, ciertos factores podrían actuar dificultando la elaboración del duelo $8,12,14-18,25$ (Tabla II), y otros potenciar factores protectores que puedan prevenir complicaciones $^{5,8,12-14,17,25-30}$.

\section{Tipo de duelos en la situación de la COVID-19}

Algunos autores proponen distintos tipos de duelo de acuerdo con la vivencia COVID-1914,15,22,31,32:

- Wallace (2020) distingue entre: a) duelo anticipado, b) duelo desautorizado, c) duelo normalizado, y d) duelo complicado.

- Maarten (2020): a) duelo resiliente, b) duelo complicado, c) duelo complicado comórbido.

- Nickerson 2020: a) duelo resiliente, b) duelo complicado, c) duelo complicado y trastorno de estrés postraumático, d) trastorno de estrés postraumático.

Selman 2020 (fallecidos en UCI): a) duelo normalizado, b) trastorno de duelo prolongado, c) trastorno de estrés postraumático, d) depresión.

Tabla I. Circunstancias del duelo por muerte por COVID-19.

No anticipación de los acontecimientos

Falta de tiempo para anticipar duelo y activar recursos personales y estrategias eficaces

Culpa por contagio, abandono, no despedirse

Desinformación o información confusa de cómo han sucedido los hechos

Desenlace rápido e inesperado

Soledad, falta de presencia, contacto y apoyo

sociofamiliar

Confinamiento

Estresores concurrentes de incertidumbre respecto a factores laborales, económicos y pérdida de la salud. Tiempo de espera para realizar los ritos o limitación de aforo. No acceso a los restos mortales del ser querido
Tabla II. Factores de riesgo específicos de duelo por la COVID-19.

1. Pérdida súbita e imposibilidad de estar o ver al fallecido

2. Creencia de que su familiar no ha recibido los cuidados que necesitaba

3. Percepción de soledad del enfermo, por no poder acompañarle los familiares

4. Confinamiento, ausencia de apoyo y ritos

5. Presencia de malestar emocional grave, reacciones de rabia, amargura y culpabilidad muy intensas

6. Problemas de salud mental previos

7. Dificultad para autorregular el impacto emocional, desbordamiento

\section{Atención al duelo por la COVID-19}

En la guía clínica SECPAL, el resumen de evidencia ${ }^{8,33}$, siguiendo los criterios de calidad de la metodología AGREE, realiza unas recomendaciones clínicas en la atención al duelo que se recogen en la Tabla III.

\section{Factores que pueden ser iatrogénicos en la atención al duelo por la COVID-19}

Algunos autores señalan la importancia de actuar evitando el daño secundario en la atención al duelo de enfermo y familiares $8,10,17,19,22,34-40$. Estos factores iatrogénicos son:

Escasez de procesos comunicativos y de atención que ayuden a todos los familiares (sin excluir niños y adolescentes, personas mayores o con problemas de salud mental o física) a ir adaptándose a la situación de incertidumbre y complejidad.

- Falta de formación y experiencia en procesos de final de vida y del duelo.

Ignorar otros duelos con riesgo, por causas ajenas a la COVID-19.

- Patologizar el duelo.

- Llevar a cabo un protocolo rígido de actuación sin respetar el ritmo personal de cada duelo.

- Forzar al familiar a ver o estar presente con enfermo o fallecido en contra de sus deseos, sin considerar otras alternativas personales posibles.

Invalidar la diversidad de expresión y vivencia de emociones, sentimientos acciones, afrontamientos, creencias y ritos.

No valorar los recursos personales de afrontamiento.

- Basar nuestra información en rumorología.

\section{OBJETIVO}

Favorecer que cada persona elabore su duelo de la manera más natural y saludable posible, prevenir factores de riesgo e identificar a lo largo de la evolución aquellos deudos que necesiten una atención específica. 
Tabla III. Guía de Atención al duelo SECPAL: evidencia y recomendaciones. Organización y actuación.

\begin{tabular}{|c|c|}
\hline Población & Tipo de actuación \\
\hline Todos los dolientes & Información acerca del duelo y sobre los recursos disponibles \\
\hline Niños y adolescentes & $\begin{array}{l}\text { Valorar la necesidad de asesoramiento por el psicólogo del equipo de cuidados } \\
\text { paliativos o servicio especializado }\end{array}$ \\
\hline Dolientes adultos de "alto riesgo" & $\begin{array}{l}\text { Valorar la necesidad de asesoramiento psicológico o una intervención precoz por el } \\
\text { psicólogo del equipo de cuidados paliativos o servicio especializado }\end{array}$ \\
\hline $\begin{array}{l}\text { Complicaciones en el duelo } \\
\text { Trastornos asociados a la pérdida }\end{array}$ & $\begin{array}{l}\text { Atención especializada (Psicólogo de Cuidados Paliativos, Atención Primaria o Salud } \\
\text { Mental) }\end{array}$ \\
\hline $\begin{array}{l}\text { Recomendaciones generales } \\
\text { 1. Proteger a la población general } \\
\text { 2. Formar a los profesionales impl }\end{array}$ & $\begin{array}{l}\text { e intervenciones inadecuadas que les puedan producir daño (latrogenia) } \\
\text { ados sobre la muerte y el duelo }\end{array}$ \\
\hline
\end{tabular}

\section{POBLACIÓN DIANA}

Personas en duelo por fallecimiento de familiar asociado a la COVID-19 en el Hospital Universitario La Paz.

\section{METODOLOGÍA}

Se estableció un protocolo de actuación en el duelo según el cual tanto el personal sanitario como los propios familiares podían pedir atención psicológica antes o después del fallecimiento ${ }^{41}$. Dicho protocolo fue distribuido por todas las plantas y en la intranet del hospital. Se definieron varios equipos de atención desde la Unidad de Cuidados Paliativos y el servicio de Psiquiatría, Psicología Clínica y Salud Mental del Hospital Universitario La Paz, compuestos por psicólogas clínicas de los equipos de infantojuvenil, adultos-interconsulta, cuidados paliativos, psicólogos internos residentes en psicología clínica, psicóloga general sanitaria, enfermeras especialistas en salud mental, enfermeras de cuidados paliativos y el apoyo de psiquiatría. Se establecieron turnos de atención de lunes a domingo de 8 a 21 horas, en las que siempre había al menos un teléfono disponible para que los diferentes profesionales del hospital pudieran acudir. Se recordó a diario en todas las plantas, urgencias y UCI el teléfono de contacto y los criterios para contactar, y se pegaron carteles informativos por todo el hospital. También se preguntó en urgencias y $\mathrm{UCl}$ qué personas estaban en situación de últimos días para ofrecer atención a las familias.

La atención se realizó en dos momentos diferentes: a) situación previa al fallecimiento: a.1.) situación de últimos días, a.2) en el momento de la "despedida final" (si esta pudo realizarse o como alternativa por teléfono o videollamada); b) situación posterior al fallecimiento: b.1.) atención, asesoramiento o intervención individualizada a deudos tras el fallecimiento de pacientes, b.2) seguimiento.

Dado el elevado riesgo de trasmisión del SARS-CoV- $2^{42}$, el protocolo de acompañamiento de pacientes durante los primeros momentos de la epidemia solo permitía la despedida final de los pacientes tras su fallecimiento. Sin embargo, conforme fue evolucionando la situación del hospital, se fueron flexibilizando las normas al respecto y también se permitió el acompañamiento de los familiares en los momentos previos al fallecimiento cuando era posible. Consideramos como "despedida final" las situaciones en las que los familiares pudieron acompañar al paciente bien en últimas horas o bien tras el fallecimiento, ya fuera de manera presencial, por carta, audio, videollamada. Cuando "la despedida final” no era posible por ningún medio se codificó como "no despedida".

\section{RESULTADOS}

\section{Características de la muestra}

Doscientas ocho familias fueron atendidas entre el 11 de marzo y el 20 de mayo de 2020. La edad media de los fallecidos fue de 76 años, siendo los grupos de entre 65-80 y 81-90 años de edad los que más frecuentemente se atendieron. El $58 \%$ de los pacientes cuyas familias fueron atendidas eran hombres. En la Tabla IV se ofrecen más detalles de la muestra.

Durante la atención en duelo por la COVID-19 se trabajó con un promedio de 1,22 familiares, siendo la atención con un familiar la más frecuente. Respecto al grado de parentesco de los familiares atendidos, fue fundamentalmente de primer grado: hijo/a (54,4\%), cónyuge o pareja ( $20 \%$ ) y hermano/a $(2,2 \%)$. En relación con el lugar de fallecimiento del paciente y, por tanto, desde donde surge la demanda de atención para salud mental, el 40,7 \% lo hace en plantas de hospitalización, el $27,1 \%$ en boxes de urgencias, el $22,1 \%$ en planta de cuidados paliativos y el $10 \%$ en plantas UCI (Tabla V).

El tipo de atención llevada a cabo con los familiares tras el fallecimiento del paciente fue en un 52,5\% de los casos telefónica, en un 16,3\% presencial, en un 11,9\% telefónica y presencial, en un 3,1\% mediante videollamada y el 6,9\% de los casos fue considerada como no necesaria, bien por la familia o bien por el profesional. En un $62,6 \%$ de las veces se realizó apoyo, asesoramiento puntual o atención específica, mientras que en el $37,4 \%$ restante se ofreció la disponibilidad de la ayuda.

En lo referente a la posibilidad de despedida presencial del familiar en situación preexitus o exitus, el $48,9 \%$ de los 
Tabla IV. Características sociodemográficas de la muestra.

\begin{tabular}{cc}
\hline Edad (años) & $\mathbf{n}(\%)$ \\
\hline $0-18$ & $0(0)$ \\
\hline $18-44$ & $4(2,1)$ \\
\hline $45-64$ & $29(14,9)$ \\
\hline $65-80$ & $70(35,9)$ \\
\hline $81-90$ & $74(37,9)$ \\
\hline$>90$ & $18(9,2)$ \\
\hline Hombre & \\
\hline Mujer & $116(58,6)$ \\
\hline
\end{tabular}

Tabla V. Características del lugar y receptor de la atención.

\begin{tabular}{lc}
\hline \multicolumn{1}{c}{$\mathrm{N}^{\circ}$ personas atendidas por familia } & $\begin{array}{c}(\mathrm{n})(\%) \\
(\text { Media, }, \mathbf{2 2})\end{array}$ \\
\hline 1 & $149(80,5)$ \\
\hline 2 & $31(16,7)$ \\
\hline Parentesco de las personas atendidas & $5(2,7)$ \\
\hline Hijo/a & $\mathrm{n}(\%)$ \\
\hline Cónyuge/pareja & $98(54,4)$ \\
\hline Más de una opción & $36(20)$ \\
\hline Hermano/a & $28(15,6)$ \\
\hline Padre/madre & $4(2,2)$ \\
\hline Nieto/a & $3(1,7)$ \\
\hline Sobrina/o & $2(1,1)$ \\
\hline \multicolumn{1}{c}{ Lugar del fallecimiento } & $9(5)$ \\
\hline Planta & $\mathrm{n}(\%)$ \\
\hline Urgencias & $57(40,7)$ \\
\hline Paliativos & $38(27,1)$ \\
\hline UCl & $31(22,1)$ \\
\hline
\end{tabular}

familiares atendidos pudieron despedirse, frente al 31,4\% que no pudo. Las causas más importantes por las que algunos familiares no pudieron despedirse fueron: normas del hospital, familiar enfermo o sospechoso aislado en casa, familiar vulnerable inmonológicamente, familiar emocionalmente no preparado o considera innecesaria la despedida, distancia de domicilio al hospital (otras provincias) o distancia en la relación (pacientes con único familiar vivo de tercer o cuarto grado de parentesco). Por último, en relación con el tipo de duelo detectado entre los familiares atendidos, este fue inicialmente normal en el 70,6\% de las veces, mientras que un $15,9 \%$ fue clasificado como de riesgo, sin diferenciar entre alto, medio o de bajo riesgo. En un 11,5\% de las familias no se obtuvo información suficiente para identificar el tipo de duelo, principalmente porque declinaron la atención cuando se les ofreció la posibilidad (Tabla VI).

\section{DISCUSIÓN}

Los resultados de las características demográficas de la muestra atendida coinciden parcialmente con los de otros estudios, donde la prevalencia de pacientes fallecidos varones es algo mayor que en el presente estudio, mientras que el rango de edad es similar 3,22,42. De forma particular a la epidemia, la atención al duelo se lleva a cabo más frecuentemente con los hijos y en porcentaje menor con la pareja o cónyuge.

Los datos muestran que, en la mayoría de las personas atendidas, su familiar falleció en una planta de hospitalización, y un $20 \%$ en la planta de Cuidados Paliativos. Las condiciones de mayor estabilidad y coordinación presentes en las plantas no UCl podrían facilitar la predicción de la evolución del paciente y la anticipación de la pérdida de

Tabla VI. Datos sobre atención y duelo.

\begin{tabular}{|c|c|}
\hline Tipo de atención en el primer contacto & n (\%) \\
\hline Telefónica & $84(52,5)$ \\
\hline Presencial & $26(16,3)$ \\
\hline No precisa/otras & $25(15,7)$ \\
\hline Telefónica y presencial & $19(11,9)$ \\
\hline Videollamada & $5(3,1)$ \\
\hline Todas & $1(0,5)$ \\
\hline \multicolumn{2}{|l|}{ Tipo de atención posterior } \\
\hline Telefónica & $100(62,5)$ \\
\hline Todas & $21(13,1)$ \\
\hline Presencial & $10(6,3)$ \\
\hline No precisa/otras & $8(6,9)$ \\
\hline Videollamada & $5(3,1)$ \\
\hline Telefónica y presencial & $5(3,1)$ \\
\hline \multicolumn{2}{|l|}{ Nivel de atención } \\
\hline $\begin{array}{l}\text { Apoyo, asesoramiento y atención } \\
\text { especializada }\end{array}$ & $117(62,6)$ \\
\hline Disponibilidad & $70(37,4)$ \\
\hline \multicolumn{2}{|l|}{ Despedida presencial } \\
\hline Sí & $67(48,9)$ \\
\hline No & $43(31,4)$ \\
\hline No datos & $27(19,7)$ \\
\hline \multicolumn{2}{|l|}{ Tipo de duelo } \\
\hline Normalizado & $147(70,6)$ \\
\hline Riesgo & $33(15,9)$ \\
\hline No especificado & $24(11,5)$ \\
\hline Anticipado & $4(1,9)$ \\
\hline
\end{tabular}


los familiares para el fatal desenlace, incluyendo el apoyo psicológico ${ }^{14,34}$. Por otra parte, también hubo un porcentaje importante $(37 \%)$ de pacientes fallecidos en urgencias y $\mathrm{UCl}$, de donde provienen la mayoría de los familiares atendidos que no pudieron despedirse de forma presencial. Muchos de estos lo hicieron a través de videollamada o por carta.

Respecto al tipo de atención, esta ha sido principalmente mediante contacto telefónico, ya que las condiciones de confinamiento y las medidas adoptadas durante el estado de alarma no permitían el acompañamiento presencial. Este último se ha dado mayoritariamente en los casos en los que se ha realizado acompañamiento al familiar en el momento que acudían para despedirse del paciente hospitalizado en situación de últimas horas, aunque también hubo casos posteriores al fallecimiento. La mayoría de los pacientes y sus familiares aceptaron la atención en duelo, facilitándose apoyo y asesoramiento psicológico por presencia de factores de riesgo, lo q coincide con otros estudios ${ }^{8}$.

En cuanto al tipo de duelo $8,14,15,22,30,32,33$, y ante la creencia de que podíamos encontrar duelos traumáticos o con dificultades por las características asociadas a la situación de la COVID-1914,22, los datos muestran que la mayoría de las personas $(70 \%)$ presentaban duelos normalizados ${ }^{14,22}$. Aunque ha pasado poco tiempo desde la pérdida, los datos recogidos son coherentes con otros estudios ${ }^{8}$. En el seguimiento de los duelos COVID-19 atendidos después de 3 meses, solo el $10 \%$ de los deudos han requerido una intervención más prolongada. Los deudos refieren que potencian recursos personales, fortalezas, resiliencia, creatividad y realizan cambios $^{3,4}$ en sus actuaciones de afrontamiento, despedidas, rituales a nivel personal y sociofamiliar (a través de videocámaras, redes sociales, etc.). Todo ello podría amortiguar el impacto de los factores de riesgo COVID-19 y facilitar duelos normalizados aun en las peores condiciones.

Esto sería congruente con algunos estudios que hablan de la existencia de un duelo resiliente de hasta un $40 \%$ de los $\operatorname{casos}^{20,22,30}$. Sin embargo estamos en un proceso preventivo en aquellos casos considerados "de riesgo" para detectar precozmente dificultades durante el duelo ${ }^{40}$.

Por otra parte, se aconseja ${ }^{8}$ un protocolo no solo de actuación sino también de seguimiento en la atención al duelo para atender los recuerdos dolorosos que se mantienen como son, últimos momentos fríos y distantes, pérdidas significativas de enseres, entrega de restos, desinformación y falta de comunicación..., vividos en muchas ocasiones como pesadillas, con incertidumbre, desconsuelo y desesperación, así como otros factores que pueden dificultar el recorrido del duelo.

En cuanto a la "despedida final", la mitad de las familias han podido llevarla a cabo de forma presencial. Hay autores que consideran que si la muerte es anticipada, y se ha podido acompañar al ser querido durante la enfermedad hasta los últimos momentos de la vida, se puede aliviar la vivencia del duelo ${ }^{14,15}$. Sabemos que en situación de pandemia y confinamiento no es fácil favorecer la presencia, pero quizás tenemos que anticipar actuaciones y ensanchar momentos en el tiempo en la medida de lo posible.

Se observa también que podría facilitar el proceso del duelo COVID-19 el tener una buena planificación y organización para prevenir la pérdida de enseres de los pacientes y atender aspectos burocráticos de la muerte (localización del tanatorio, cadáver, documentos necesarios, etc. $)^{17}$. Asimismo, una información y comunicación efectiva con los familiares ${ }^{19}$ acerca de la situación y calidad de cuidados al enfermo ${ }^{25}$ y disponibilidad de atención psicológica ${ }^{17}$, sería otro aspecto importante para facilitar una elaboración saludable del duelo.

Consideramos que uno de los puntos fuertes de la atención en duelo ha sido la labor de dar visibilidad a la misma. La disponibilidad del protocolo de actuación en duelo por COVID-19 en la intranet del hospital, los carteles distribuidos en las diferentes unidades, así como la comunicación más informal.

El desconocimiento del duelo específico relacionado con el confinamiento y la epidemia de SARS-CoV-2, la imprevisibilidad y la no anticipación de los hechos han dificultado la previsión de un registro común llevado a cabo por los diferentes profesionales en la atención al duelo. Asimismo, las diferencias en formación de los múltiples profesionales implicados han podido limitar la capacidad de generalización de los diferentes niveles de atención realizados. Sin embargo, sí se ha mantenido un objetivo común que era la "no iatrogenia" en las actuaciones.

\section{CONCLUSIONES Y RECOMENDACIONES PARA EL FUTURO}

1. La situación excepcional por la COVID-19 que hemos vivido, con unas consecuencias para las que no estábamos preparados, ha cambiado nuestra forma de estar y vivenciar el mundo, nos han llevado a realizar cambios en todos los ámbitos de nuestra vida, incluso de nuestra cultura, ritos, forma de morir y de despedirnos de nuestros seres queridos sin el apoyo sociofamiliar acostumbrado.

2. Parece importante anticipar actuaciones y "ensanchar el tiempo", para que la familia pueda acompañar al paciente, anticipar y prepararse para la muerte, lo que puede favorecer el proceso de duelo.

3. Una buena planificación y organización para prevenir la pérdida de enseres de los pacientes y atender temas burocráticos de la muerte (localización tanatorio, cadáver...) por un lado, y una información y comunicación efectiva con los familiares, disponibilidad del apoyo psicológico, podrían ser de mucha importancia en un duelo por la COVID-19.

4. Se considera necesario formar a los profesionales implicados en la muerte y en el duelo para no causar "iatrogenia" e incluir la filosofía de cuidados paliativos en estas situaciones.

5. La experiencia muestra que la "atención al duelo" debe estar a disposición de la población general afectada con protocolos de actuación y seguimiento que incluyan valoración y atención en los diferentes niveles desde el apoyo, la prevención y la detección precoz de posibles complicaciones en el duelo y si es necesario la intervención especializada, como recomienda la Guía de Atención al Duelo en Cuidados Paliativos SECPAL. 


\section{BIBLIOGRAFÍA}

1. Radbruch L, Knaul FM, Lima L de, Joncheere C de, Bhadelia A. The key role of palliative care in response to the COVID-19 tsunami of suffering. Lancet. 2020;395:1467-9.

2. Blake H, Bermingham F, Johnson G, Tabner A. Mitigating the psychological impact of COVID-19 on healthcare workers: a digital learning package. Int J Environ Res Public Health. 2020;17:2997.

3. Liu S, Yang L, Zhang C, Xiang Y-T, Liu Z, Hu S, et al. Online mental health services in China during the COVID-19 outbreak. Lancet Psychiatry. 2020;7:e17-8.

4. Breen LJ. Grief loss and the COVID-19 pandemic. Aust J Gen Pract. 2020;49.

5. Etkind SN, Bone AE, Lovell N, Cripps RL, Harding R, Higginson IJ, et al. The Role and Response of Palliative Care and Hospice Services in Epidemics and Pandemics: A Rapid Review to Inform Practice during the COVID-19 Pandemic. J Pain Symptom Manage. 2020;60:e31-e40.

6. Morris SE, Moment A, Thomas J deLima. Caring for Bereaved Family Members During the COVID-19 Pandemic: Before and After the Death of a Patient. J Pain Symptom Manage. 2020;60:e70-e74.

7. Castle J, Phillips WL. Grief Rituals: Aspects That Facilitate Adjustment to Bereavement. J Loss Trauma. 2003;8:41-71.

8. Lacasta Reverte MA, Sanz Llorente B, Soler Saíz MC, Yelamos Agua C, de Quadras Roca S, Gándara del Castillo A. Atención al duelo en Cuidados Paliativos: Guía clínica y Protocolo de actuación. Madrid: Síosí Punto Gráfico; 2014.

9. Lundorff $M$, Holmgren H, Zachariae R, Farver-Vestergaard I, O'Connor M. Prevalence of prolonged grief disorder in adult bereavement: A systematic review and meta-analysis. J Affect Disord. 2017;212:138-49.

10. Eisma MC, Boelen PA, Lenferink LIM. Prolonged grief disorder following the Coronavirus (COVID-19) pandemic. Psychiatry Res. 2020;288:113031.

11. Chew NWS, Lee GKH, Tan BYQ, Jing M, Goh Y, Ngiam NJH, et al. A multinational, multicentre study on the psychological outcomes and associated physical symptoms amongst healthcare workers during COVID-19 outbreak. Brain Behav Immun. 2020;88:559-65.

12. Curtis JR, Kross EK, Stapleton RD. The Importance of Addressing Advance Care Planning and Decisions about Do-Not-Resuscitate Orders During Novel Coronavirus 2019 (COVID-19). JAMA. 2020;323:1771-2.

13. Hospital de Manacor. Guía de apoyo clínico en toma de decisiones, control sintomático y acompañamiento en situaciones COVID-19 [Internet]. SECPAL; 30 de marzo de 2020 [citado 14 de junio de 2020]. Disponible en: http: / /www.secpal.com//Documentos/Blog/VA\%CC\%82\%C2\%BA\%202.0-GUIA\%20DE\%20 APOYO\%20TD-CS-ACOM-COVID19-HM.pdf.pdf.pdf.pdf.

14. Wallace CL, Wladkowski SP, Gibson A, White P. Grief During the COVID-19 Pandemic: Considerations for Palliative Care Providers. J Pain Symptom Manage. 2020;60:e70-e76.

15. Selman LE, Chao D, Sowden R, Marshall S, Chamberlain C, Koffman J, Bereavement support on the frontline of COVID-19: Recommendations for hospital clinicians. J Pain Symptom Manage. 2020;60:e81-e86.

16. Rubin R. Challenge Trials-Could Deliberate Coronavirus Exposure Hasten Vaccine Development? JAMA. 2020. DOI: 10.1001/ jama.2020.9881.

17. Zhai Y, Du X. Loss and grief amidst COVID-19: A path to adaptation and resilience. Brain Behav Immun. 2020;87(80-1.

18. Cao W, Fang Z, Hou G, Han M, Xu X, Dong J, et al. The psychological impact of the COVID-19 epidemic on college students in China. Psychiatry Res. 2020;287:112934.

19. Mayland CR, Harding AJE, Preston N, Payne S. Supporting Adults Bereaved Through COVID-19: A Rapid Review of the Impact of
Previous Pandemics on Grief and Bereavement. J Pain Symptom Manage. 2020;60:e33-e39.

20. Bonnano GA. Loss, trauma and human resilience: Conceptual and empirical connections and separateness. Am Psychol. 2004;59:20-8.

21. Litz BT. Has Resilience to Severe Trauma Been Underestimated? Am Psychol. 2005;60:262.

22. Eisma MC, Lenferink LIM, Chow AYM, Chan CLW, Li J. Complicated grief and post-traumatic stress symptom profiles in bereaved earthquake survivors: a latent class analysis. Eur J Psychotraumatology. 2019;10:1558707.

23. Boelen PA, van de Schoot R, van den Hout MA, de Keijser J, van den Bout J. Prolonged Grief Disorder, depression, and posttraumatic stress disorder are distinguishable syndromes. J Affect Disord. 2010;125:374-8.

24. Lobb EA, Kristjanson LJ, Aoun SM, Monterosso L, Halkett GKB, Davies A. Predictors of Complicated Grief: A Systematic Review of Empirical Studies. Death Stud. 2010;4:673-98.

25. Sutherland AE, Stickland J, Wee B. Can video consultations replace face-to-face interviews? Palliative medicine and the Covid-19 pandemic: rapid review. BMJ Supportive Palliative Care. 2020;10:271-5.

26. Corr CA, Doka KJ. Current Models of Death, Dying, and Bereavement. Crit Care Nurs Clin. 1994;6:545-52.

27. Stroebe MS, Schut H. Meaning making in the dual process model of coping with bereavement. En: Neimeyer RA, editor. Meaning reconstruction \& experience of loss. American Psychological Association; 2001. p. 55-73.

28. Stroebe M, Finkenauer C, Wijngaards-de Meij L, Schut $H$, van den Bout J, Stroebe W. Partner-oriented self-regulation among bereaved parents: The costs of holding in grief for the partner's sake. Psychol Sci. 2013;24:395-402.

29. Mancini AD, Bonanno GA. Resilience in the face of potential trauma: Clinical practices and illustrations. J Clin Psychol. 2006;62:971-85.

30. Tamiolaki A, Kalaitzaki AE. "That which does not kill us, makes us stronger": COVID-19 and Posttraumatic Growth. Psychiatry Res. 2020;289:113044.

31. Olff M, Bakker A, Frewen P, Aakvaag H, Ajdukovic D, Brewer D, et al. Screening for consequences of trauma - an update on the global collaboration on traumatic stress. Eur J Psychotraumatology. 2020;11:1752504.

32. Association AP. Diagnostic and statistical manual of mental disorders (DSM-5®). American Psychiatric Pub; 2013.

33. Schut H, Stroebe MS, van den Bout J, Terheggen M. The efficacy of bereavement interventions: Determining who benefits. En: Handbook of bereavement research: Consequences, coping, and care. Washington, DC, US: American Psychological Association; 2001. p. 705-37.

34. Costantini M, Sleeman KE, Peruselli C, Higginson IJ. Response and role of palliative care during the COVID-19 pandemic: A national telephone survey of hospices in Italy. Palliat Med. 2020;34:889-95.

35. Comtesse H, Vogel A, Kersting A, Rief W, Steil R, Rosner R. When does grief become pathological? Evaluation of the ICD-11 diagnostic proposal for prolonged grief in a treatment-seeking sample. Eur J Psychotraumatology. 2020;11:1694348.

36. Boelen PA, Lenferink LIM. Comparison of six proposed diagnostic criteria sets for disturbed grief. Psychiatry Res. 2020;285:112786.

37. Wakefield JC. Should Prolonged Grief Be Reclassified as a Mental Disorder in DSM-5?: Reconsidering the Empirical and Conceptual Arguments for Complicated Grief Disorder. J Nerv Ment Dis. 2012;200:499-511.

38. Horesh D, Brown AD. Traumatic stress in the age of COVID-19: A call to close critical gaps and adapt to new realities. Psychol Trauma Theory Res Pract Policy. 20200409;12:331. 
39. Kristensen $P$, Weisæth $L$, Heir T. Bereavement and Mental Health after Sudden and Violent Losses: A Review. Psychiatry Interpers Biol Process. 2012;75:76-97.

40. Rogers J, et al. Psychiatric and neuropsychiatric presentations associated with severe coronavirus infections: a systematic review and meta-analysis with comparison to the COVID-19 pandemic. Lancet Psychiatry. 2020:7:611-27.
41. Unidad de Cuidados Paliativos, Servicio de Psiquiatría, Psicología Clínica y Salud Mental. Protocolo de atención a pacientes en situación de gravedad o últimos días y éxitus en la crisis del COVID-19. Hospital La Paz; 2020.

42. Borobia AM, Carcas AJ, Arnalich F, Álvarez-Sala R, Monserrat-Villatoro J, Quintana M, et al. A Cohort of Patients with COVID-19 in a Major Teaching Hospital in Europe. J Clin Med. 2020;9:1733. 